\title{
Construction and diversity analysis of a murine IgE phage surface display library
}

\author{
LI Zong Dong, Ming YEH ${ }^{1}$ \\ Shanghai Institute of Cell Biology, Chinese Academy of \\ Sciences, Shanghai 200031, China
}

\begin{abstract}
To make further investigation of the IgE antibody repertoire in Trichosanthin (TCS) allergic responses, a murine IgE phage surface display library was constructed $\left(3.0 \times 10^{5}\right.$ independent clones). We first constructed the $\mathrm{V} \varepsilon$ cDNA library $\left(4.6 \times 10^{5}\right.$ independent clones $)$ and $\mathrm{V} \kappa$ cDNA library $\left(3.0 \times 10^{5}\right.$ independent clones). Then, the $\mathrm{V} \varepsilon$ and $\mathrm{V} \kappa$ gene segments were amplified from both libraries by PCR respectively, and assembled into Fab fragment by SOE PCR. The phage library containing Fabs was thus constructed. The diversity of $\mathrm{V} \varepsilon$ from this library was analyzed and proved. Fab clones with high specificity to TCS have been screened out.
\end{abstract}

Key words: Phage surface display library, trichosanthin, murine IgE antibody.

\section{INTRODUCTION}

In our previous study of $\mathrm{V} \varepsilon$ and $V \kappa$ from four anti-TCS IgE hybridomas established in our laboratory, we found $\mathrm{V} \kappa$ from all four clones used fragments from the same germline gene family $\mathrm{V} \kappa 21$, and a bias in the use of Jk1 gene fragment was also observed. On the other hand, the gene usage of $\mathrm{VH}$ was quite diverse. We speculated that in IgE responses to TCS, the light chain may play a more important role in specific binding to allergenic determinant on TCS[1]. However, due to the limitation of hybridoma technology, it is difficult to achieve a large number of anti-TCS IgE clones. The recent advent of phage surface display antibody library

1. Corresponding author, e-mail: imceng@server.shcnc.ac.cn 
technology provides us the opportunity to study anti-TCS IgEs on a bigger capacity. We constructed a human IgE phage antibody library from normal human peripheral blood lymphocytes and screened out two clones[2]. However their affinity to TCS was low, and the diversity of the library was not satisfactory, thus it could hardly provide us more useful informations about the IgE recognition of TCS.

In this study, we used TCS-immunized mice. $\mathrm{V} \varepsilon$ segments and $\mathrm{V} \kappa$ segments were obtained through RT-PCR. First a $\mathrm{V} \varepsilon$ cDNA library and a V $\kappa$ cDNA library were constructed separately. $\mathrm{V} \varepsilon$ and $\mathrm{V} \kappa$ fragments amplified from the two libraries were assembled into a large fragment by SOE PCR. The expression vector was reconstructed for the expression of Fab fragments. And factors which potentially influence the results of SOE PCR and the diversity of the library were analyzed at the same time. Based on these work, we constructed a phage surface display murine Fab library, and its diversity and the antigen specificity of the expressed clones were analyzed.

\section{MATERIALS AND METHODS}

\section{Plasmids and bacterial strains}

Vector pHEN1, plasmid pSW1 Fab (D1. 3) and the E. coli strain TG1 were all kindly provided by Dr G. Winter, MRC, Cambridge. Plasmid pBluescript and bacterial strain XL1-Blue were from our laboratory.

\section{Primers}

Primers were designed according to Orlandi[3]:

VH Back:

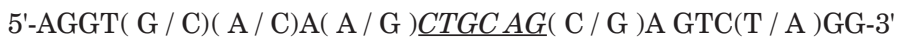

PstI

VH Back SfiI:

5'-CAACTGCGGCCCAGCCGGCCATGGCCCAGGT(G/C)(A/C)A(A/G)CTGCAG SfiI

(C/G) AGTC(T/A)GG-3'

JH For:

5'-TGAGGAGAGACGGTGACCGTGGTCCCTTGGCCCCAG-3'

BstEII

Reverse JH For(rJH)

5'-CTGGGGCCAAGGGACCACGGTCACCGTCTCCTCA-3'

VK Back:

5'-AGCGATGGCCGACATTGAGCTCACCCAGTCTCAA-3'

ScaI

Reverse VK Back(rVK):

5'-GGAGACTGGGTGAGCTCAATGTC-3' 


\section{Li ZD and M Yeh}

JK For:

5'-GTTTGATCTCGAGCTTGGTGCC-3'

XhoI

CK For NotI

5'-GACTTGCGGCCGCAGACTCTCCCCTGTTGAAGGCTCTT-3'

NotI

To ensure the specific amplification of $\mathrm{V} \varepsilon$ segments, a $\mathrm{C} \varepsilon$ primer was designed according to the constant region of IgE antibody for reverse transcription and PCR amplification.

$\mathrm{C} \varepsilon$ For primer:

5'-GAAGCAGTGCCTTTACAGGGCT-3'

\section{RT-PCR amplification of $\mathrm{V} \varepsilon$ and $\mathrm{V} \kappa$ segments}

RNA was extracted from the mesenteric lymph node of C57BL/6J mice[4], which had been immunized intraperitoneally at a dose of $5 \mu \mathrm{g}$ of TCS (Jin San Pharmaceutical Factory) for 3 times. Appropriate primers were used for different amplification. $\mathrm{C} \varepsilon$ primer and Jk primer were used for first strand cDNA synthesis separately. For $\mathrm{V} \varepsilon$ segments, the cDNA sample was first amplified with $\mathrm{C} \varepsilon$ primer and VH primer and the resulting PCR products were used as template in the second round PCR amplification with primer $\mathrm{VH}$ and JH.

PCR was performed in a total volume of $50 \mu$ l, containing $5 \mu \mathrm{l}$ of $10 \times$ reaction buffer, $2 \mu \mathrm{l}$ of $5 \mathrm{mM}$ dNTPs, $1 \mu 1$ of each appropriate back and forward Primer $(25 \mathrm{pmol} / \mu \mathrm{l}), 1 \mu \mathrm{l}$ Taq DNA polymerase $\left(2 \mu \mathrm{g} / \mu 1\right.$, Promega), $3 \mu \mathrm{l} \mathrm{cDNA}$ sample and $37 \mu 1 \mathrm{ddH}_{2} \mathrm{O}$. The PCR was run for 35 cycles $\left(94{ }^{\circ} \mathrm{C} 1 \mathrm{~min}, 50{ }^{\circ} \mathrm{C} 1 \mathrm{~min}, 72{ }^{\circ} \mathrm{C} 1 \mathrm{~min}\right)$.

\section{Construction of $\mathrm{V} \varepsilon$ and $\mathrm{V} \kappa$ cDNA library[5]}

$\mathrm{V} \varepsilon$ and $\mathrm{V} \kappa$ segments were ligated into pBluescript (SmaI) by blunt end ligation, which could decrease the possible loss of fragments in comparison with the method of restriction enzyme digestion. The subsequent cloning was carried out as routine. The ligation products were used to transform E. coli XL1-Blue through electroporation. The transformants were plated and counted to determine the capacity of the library. Three clones were randomly chosen from the libraries, two from $\mathrm{V} \varepsilon$ cDNA library and one from $\mathrm{V} \kappa$ eDNA library. They were sequenced to check whether they belonged to immunoglobulin genes.

\section{Reconstruction of the expression vector}

For convenient expression of Fab fragment of mouse, we introduced $\mathrm{C} \kappa$ segment into pHEN1. CH1 Fablinker fragment was amplified from pSW1D1.3 plasmid with primer rJH and rVk and digested by XhoI and NotI. After recovered and purified by agarose gel electrophoresis, the $\mathrm{C} \kappa$ fragment was ligated into pHEN1 (XhoI, NotI) to construct an expression vector pHEN1C $\kappa$.

\section{Assembly of $V \varepsilon$ and $V \kappa$ segments by SOE PCR[6]}

All fragments used for SOE PCR should be purified through low melting point agarose gel electrophoresis. The SOE PCR included two steps: First, a $40 \mu$ reaction mixture was prepared, which contained $4 \mu \mathrm{l}$ of $10 \times$ reaction buffer, $2 \mu \mathrm{l}$ of $5 \mathrm{mM}$ dNTPs, each sample $250 \mathrm{ng}$ at least, and $2 \mu \mathrm{l}$ Taq DNA polymerase $\left(2 \mathrm{u} / \mu 1\right.$, Promega). The reaction cycle was $94{ }^{\circ} \mathrm{C} 1 \mathrm{~min}$ and $75{ }^{\circ} \mathrm{C}$ 3 min. After 7 cycles, the temperature was held at $72{ }^{\circ} \mathrm{C}$ and another mixture was added. This mixture was $10 \mu \mathrm{l}$ in volume, including $50 \mathrm{pmol}$ each appropriate primer and $1 \mu \mathrm{l}$ of $10 \times$ reaction buffer. After thoroughly mixing the second cycling was started immediately. The cycle was $94{ }^{\circ} \mathrm{C}$ $1 \mathrm{~min}, 50{ }^{\circ} \mathrm{C} 1 \mathrm{~min}, 72{ }^{\circ} \mathrm{C} 2 \mathrm{~min}$. After 20 cycles, the reaction mixture was held at $72{ }^{\circ} \mathrm{C}$ for 5 min. (Fig 1) 


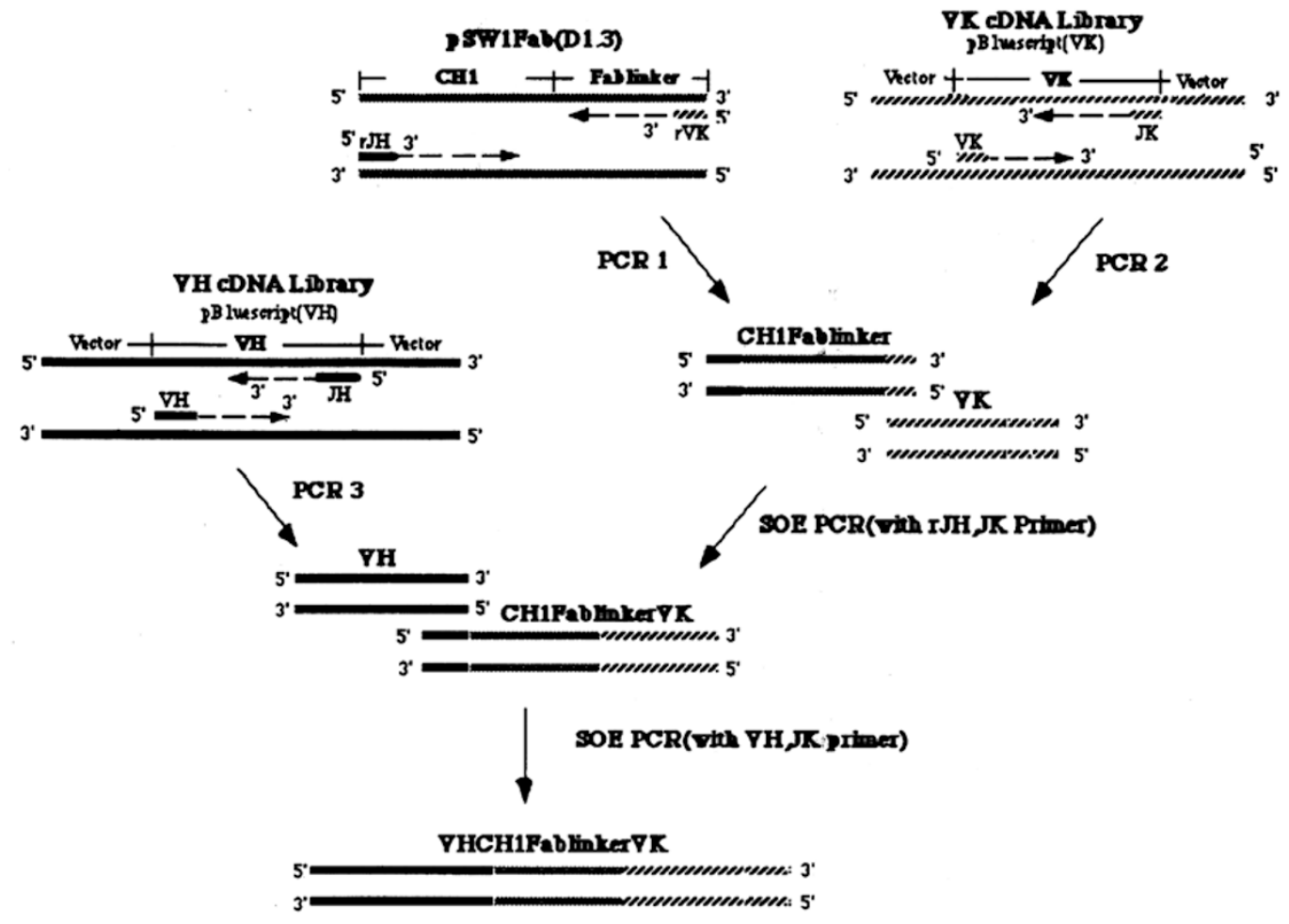

Fig 1. The process of assembly of the antibody fragment by SOE PCR

Construction of phage surface display library and analysis of the diversity of $V \varepsilon$ segments

The fragments assembled through SOE PCR were digested with SfiI and XhoI, and recovered through low melting point gel electrophoresis. Ligation products were used to transform XL1-Blue by electroporation. Ten clones were selected from two libraries ( $\mathrm{V} \varepsilon$ cDNA library and phage library) at random. The $\mathrm{V} \varepsilon$ fragments were amplified from these clones and digested with HaeIII, RsaI and MspI (SABC). The products were loaded onto $8 \%$ PAGE gel for electrophoresis. Band patterns from electrophoresis would indicate the differences among these $\mathrm{V} \varepsilon$ segments.

\section{Screening of the anti-TCS Fab clones from the phage antibody library}

\section{1) Generation of phage antibody}

The phage antibody library transformed bacteria were inoculated into $2 \times$ TY broth containing $100 \mu \mathrm{g} / \mathrm{ml} \mathrm{Amp}$ and $1 \% \mathrm{Glu}$ and grown at $37{ }^{\circ} \mathrm{C}$ with shaking. After centrifugation and resuspending, helper phage (M13-K07) were added and cultured again. The phage particles were repeatedly precipitated with PEG. After centrifugation the phage particles were resuspended in $3 \mathrm{ml}$ PBS-1 $\%$ BSA. $10^{12}$ phage particles in suspension were added to TCS $(20 \mu \mathrm{g} / \mathrm{ml})$ coated and $1 \%$ gelatin blocked plate (Cel-cult, six wells). They were held in room temperature for $2 \mathrm{~h}$. The plates were washed thoroughly first with PBS-0.1\% Tween and then with PBS. The phage particles were eluted 
with $100 \mathrm{~m} M$ triethylamine. The eluates were neutralized with $1.0 M$ Tris-HCl (Ph 7.4). They were used to transform XL1- Blue at standstill for $45 \mathrm{~min}$ and then plated on 2YT-Amp-Glu plates.

2) ELISA analysis of the phage antibody and the soluble Fab:

ELISA for phage antibody: Clones grown on the plates were picked up and cultured in 2YTAmp-Glu overnight till the $\mathrm{OD}_{600}$ up to 0.5. Helper phages were added and cultured with shaking again. After centrifugation and resuspending, the phage suspension was subjected to ELISA. The ELISA plate was separately coated with TCS, OVA (Sigma), and BSA (Dong Fong Reagent Co). Phage suspension was added. After washing, anti- M13 antiserum (prepared in our lab) and peroxidase labeled goat anti- rabbit Ig antibodies (BioRad) were added in turn. The OD value was read with a ELISA Reader (Bio-Tek).

ELISA for soluble Fab: Phages were used to transfect E. coli HB2151, and cultured in 2YTAmp till the $\mathrm{OD}_{600}$ up to 0.9. Then IPTG was added and they were cultured for another $18 \mathrm{~h}$. After centrifugation the supernatant was used for routine ELISA. Test antigens were coated as above.

\section{RESULTS}

\section{RT-PCR amplification of $\mathrm{V} \varepsilon$ and $\mathrm{V} \kappa$ segments}

Results from electrophoresis showed that the $\mathrm{V} \varepsilon$ and $\mathrm{V} \kappa$ bands were of expected size, with $\mathrm{V} \varepsilon$ about 350 bp and $\mathrm{V} \kappa$ about 320 bp. (Fig 2)

Fig 2. The electrophoresis result of $\mathrm{V} \varepsilon$ and $\mathrm{V} \kappa$ PCR amplification

1. $\lambda /$ HindIII + EcoRI

2. PCR amplification of $\mathrm{V} \varepsilon$ (left arrow)

3. PCR amplification of $\mathrm{V} \kappa$ (right arrow)

4. 500bp fragment

5. $800 \mathrm{bp}$ fragment

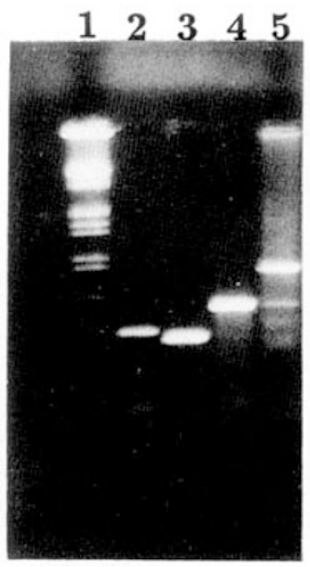

The capacity of the two cDNA libraries were: $4.6 \times 10^{5}$ independent clones for $\mathrm{V} \varepsilon$ cDNA library and $3.0 \times 10^{5}$ clones for $\mathrm{V} \kappa$ cDNA library. Results from sequencing of the three cDNA clones from the two libraries showed that all of them belonged to immunoglobin genes. The putative germ-line genes used by V, D, J gene segments of Clone VH2, VH6 and V $\kappa 3$ were shown in Tab 1. 
Murine anti-TCS IgE phage library

\section{The identification of vector $p H E N 1 C \kappa$}

To determine whether the $\mathrm{C} \kappa$ insertion in the vector pHEN1 was correct, XhoI and NotI were used to digest the constructed vector $\mathrm{pHEN} 1 \mathrm{C} \kappa$. The electrophoresis results showed there was a band about $300 \mathrm{bp}$ which was of the expected size of $\mathrm{C} \kappa$ fragment (Fig 3).

Tab 1. The putative germ-line gene usage of by V, D, J gene segments of Clone VH2, VH6 and V $\kappa 3$

\begin{tabular}{lccc}
\hline & VH2 & VH6 & V 3 \\
\hline V gene family & J558 & Q52 & V 10 \\
V gene segment & $122 \mathrm{~b}$ & Q52 & V $\kappa 10$ \\
D gene segment & DSP2.7 & DSP2.5 & \\
J gene segment & JH2 & JH4 & JK1 \\
\hline
\end{tabular}

3. $V \varepsilon$ and $V \kappa$ fragments assembled by SOE PCR

First, V $\kappa$ was assembled with CH1Fablinker. The products were about $800 \mathrm{bp}$ as expected. The resulting products, CH1 Fab linker V $\kappa$ fragments, were then assembled with $\mathrm{V} \varepsilon$ fragments. The products were about $1.1 \mathrm{~kb}$ (Fig 4).

\section{3}

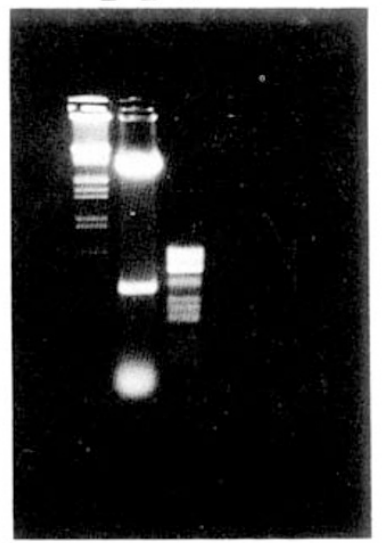

Fig 3. The identification of expression vector

1. Marker $(\lambda /$ HindIII + EcoRI $)$

2. pHEN1C $\kappa$ (XhoI, NotI) arrow

3. pBR322 (HaeIII)

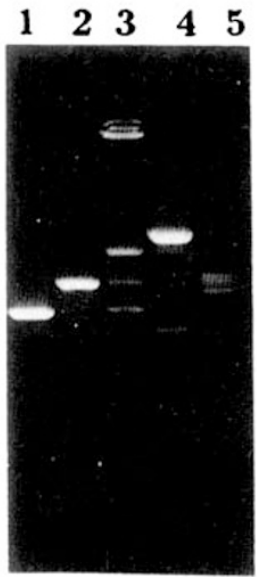

Fig 4. Electrophoresis results of SOE PCR

1. PCR amplification of $\mathrm{V} \kappa$

2. PCR amplification of $\mathrm{CH} 1$ Fablinker

3. SOE PCR amplification of $\mathrm{V} \kappa-\mathrm{CH} 1$ Fablinker (left arrow)

4. SOE PCR amplification of $\mathrm{V} \varepsilon-\mathrm{CH} 1$

Fablinker-V $\kappa$ (right arrow)

5. pBR322 (HaeIII) 
4. Diversity analysis of the $V \varepsilon$ fragments from cDNA library and phage library

VH Back and JK For primer were used to amplify $\mathrm{V} \varepsilon$ fragments of the clones randomly chosen from both libraries. The $\mathrm{V} \varepsilon$ fragments were digested with restriction enzyme HaeIII, MspI and RsaI separately. The digested products were loaded onto $8 \%$ PAGE gel. Electrophoresis results showed the differences among the $\mathrm{V} \varepsilon$ segements, which suggested that the $\mathrm{V} \varepsilon$ in phage library still kept the diversity as it was in vivo. (Fig 5, Fig 6).

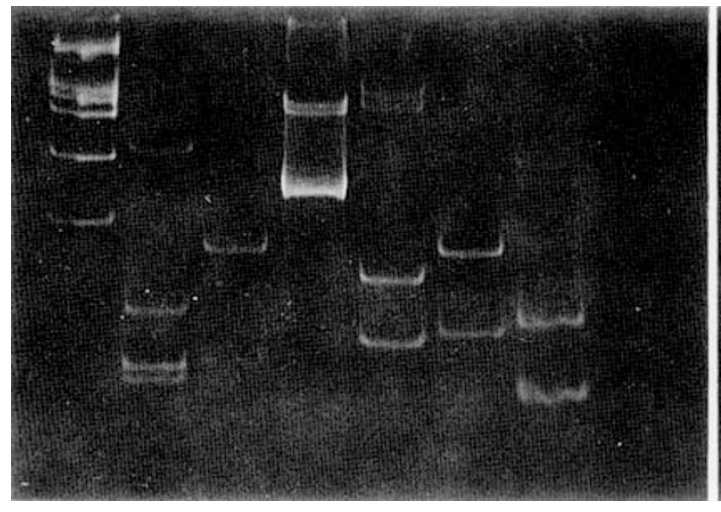

Fig 5. Diversity analysis of the $\mathrm{V} \varepsilon$ from cDNA library

All the clones are randomly selected from $\mathrm{V} \varepsilon$ cDNA library.

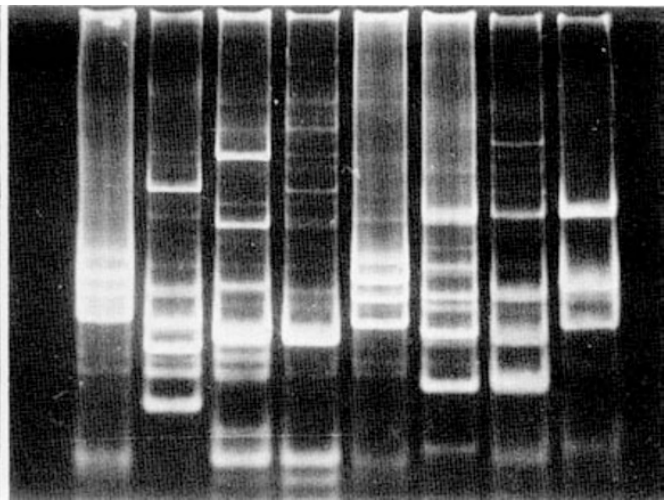

Fig 6. Diversity analysis of the $\mathrm{V} \varepsilon$ from phage library All the clones are randomly selected from phage library.

\section{ELISA results:}

Two phage antibody clones, Fab2 and Fab7, were chosen from library screening. Both phage-ELISA and soluble Fab-ELISA all showed the TCS-specific binding of the two Fab clones, with Fab 7 with higher binding specificity. (Tab 2, Tab 3)

Tab 2. Antigen specificity of phage antibody

\begin{tabular}{cccc}
\hline $\mathrm{O}_{450}(\mathrm{X} \pm \mathrm{SD})$ & TCS & OVA & BSA \\
\hline Fab2 & $0.68 \pm 0.02$ & $0.19 \pm 0.03$ & $0.16 \pm 0.02$ \\
Fab7 & $1.36 \pm 0.04$ & $0.15 \pm 0.04$ & $0.11 \pm 0.03$ \\
Control(vector) & $0.10 \pm 0.02$ & $0.11 \pm 0.01$ & $0.06 \pm 0.01$ \\
\hline
\end{tabular}

Tab 3. Antigen specificity of soluble Fab

\begin{tabular}{cccc}
\hline O.D $_{450}(\mathrm{X} \pm \mathrm{SD})$ & TCS & OVA & BSA \\
\hline Fab2 & $0.54 \pm 0.02$ & $0.32 \pm 0.03$ & $0.39 \pm 0.03$ \\
Fab7 & $0.97 \pm 0.02$ & $0.37 \pm 0.02$ & $0.47 \pm 0.03$ \\
Control(vector) & $0.18 \pm 0.02$ & $0.16 \pm 0.01$ & $0.17 \pm 0.02$ \\
\hline
\end{tabular}


Murine anti-TCS IgE phage library

\section{DISCUSSION}

\section{cDNA library construction}

That TCS alone (without using adjuvant) can induce specific IgE responses has been proved in our laboratory[7]. In the lymphocyte repertoire, B cell clones producing TCS-specific antobodies including specific IgE were surely enriched. The $\mathrm{V} \varepsilon$ and $\mathrm{V} \kappa$ cDNA library derived from the repertoire would accordingly reflect the distribution of the different B cell clones. After antigen selection (library screening) it is possible to probe the in vivo IgE responses from the in vitro constructed repertoire (phage antibody library). The reason that we first constructed the two cDNA libraries instead of assembling $\mathrm{V} \varepsilon$ and $\mathrm{V} \kappa$ directly from cDNA PCR products is due to our previous experiences that the specific amplification bands were seldom acquired and non-specific amplification was very strong. In order to keep the diversity more close to the status in vivo, the fragments were ligated into pBluescript through blunt end ligation. Otherwise some segments would most probably be lost during restriction enzyme digestion for cloning. Two clones and one clone have been randomly selected from $\mathrm{V} \varepsilon$ and $\mathrm{V} \kappa$ library respectively. Their sequences showed that all of them were from immunoglobulin $\mathrm{V}$ region genes (data not shown). Thus, the RT- PCR amplification products were specific for Ig V region genes. To ensure specific amplification of $\mathrm{V} \varepsilon$ fragments nested PCR were used to guarantee that the amplified fragments were $\mathrm{V} \varepsilon$.

\section{Assembly of antibody fragment by SOE PCR}

So far as the method of assembly of VH and VL into Fab fragment, specific enzyme digestion and then cloning into vectors are frequently used in the papers. The method is relatively simple and direct. However, it is not uncommonly to find that limited clones are obtained. In condideration of preserving the diversity as much as possible, SOE PCR seems to be a method of choice, because it reduces the loss during enzyme digestion. To preserve the diversity of $\mathrm{V} \varepsilon$ fragment, a degenerate primer $(\mathrm{V} \varepsilon$ Back) was used in $\mathrm{V} \varepsilon$ amplification. However, it caused a number of nonspecific amplification during SOE PCR. Therefore, we had to construct the cDNA library of $\mathrm{V} \varepsilon$ and $\mathrm{V} \kappa$ respectively. Since more plasmids could be obtained from cDNA library of $\mathrm{V} \kappa$ and $\mathrm{V} \varepsilon$ and more $\mathrm{V} \kappa$ and $\mathrm{V} \varepsilon$ fragments could be got to be used as PCR templates, thus the PCR amplification rounds could be reduced. Furthermore it is easy to get more $\mathrm{V} \varepsilon$ and $\mathrm{V} \kappa$ fragments as templates of SOE PCR, which in turn provides sufficient amount of SOE PCR products for library construction and need not to do another round of amplification of SOE PCR products, thus reducing error synthesis[8].

In this study we found that the overlap length of the primer (Forward and Reverse primer) should be over 30 base pairs. If the overlap is more than 30 base pairs, the annealing and extension temperature in the first step of assembly could be set at $75{ }^{\circ} \mathrm{C}$. It is higher than usual, thus also reducing nonspecific amplification. 


\section{The diversity of the library}

The diversity of the library is of prior importance for further study of the TCSspecific IgE responses. From Fig 5 and 6, it indicated that 6 clones randomly picked up from $\mathrm{V} \varepsilon$ cDNA library and 8 clones randomly picked up from phage Fab library were all different clones. Hence it proved there was no obvious loss of diversity in the two libraries and highly specific antibody clones could be screened out. Although the capacity of the library seems not big enough, $3 \times 10^{5}$ clones, however the frequency of IgE-producing B cells is very small. It was reported, in human PBL IgE- producing B cells frequently only account for $0.001 \%-0.01 \%$ [9]. In this sense the library constructed may probably provide sufficient information to analyse TCS-specific IgE responses. However, it should point out that during the construction of library, some technical factors may influence the diversity, such as errors from PCR amplification, enzyme digestion, and transformation efficiency. We should be aware of them during library construction.

The most straight forward method to study diversity is sequencing, however, it is labor intensive. The present enzyme digestion method using 3 restriction enzymes with 4 bp recognition site provides an alternative. The fine digestion patterns shown in electrophoresis indicate it could be used instead.

\section{Phage Fab and soluble Fab}

In Tab 3 the ELISA values of soluble Fabs are not as specific and higher as that of the phage Fabs. Since our vector is not high expression vector, the expression products in the culture supernatant are not in high concentration. This may be one of the reasons. Futhermore, in ELISA used for determination of soluble Fabs, after adding soluble Fab only one second layer antibody (Fab- specific) is added. While in phage ELISA, the Fabs are expressed on the phage particles and both second layer antibody, rabbit anti-M13 antiserum and, third layer antibody, goat anti-rabbit Ig antibodies, are used, so the amplification is bigger. This may also explain the differences.

\section{ACKNOWLEDGEMENTS}

The work was supported by the Director Grant of Shanghai Institute of Cell Biology, Chinese of Academy of Sciences, and also supported by the World Laboratory. We are most grateful to the generous and great help of Dr. Greg Winter in Cambridge, England.

\section{REFERENCES}

[1] Wang Y, Yeh M. Molecular Characteriation of the V regions of four IgE antibodies specific for Trichosanthin. Immunology 1996; 89(3):316-23. 


\section{Murine anti-TCS IgE phage library}

[2] Zan Hong, Ming Yeh. Construction of normal human IgE phage antibody library and the screening of the anti-trichosanthin IgE. Cell Research 1996; 6:1-9.

[3] Orlandi R, Gussow DH, Jones PT, Winter G. Cloning immunoglobulin variable domain for expression by the Polymerase Chain Reaction. PNAS 1989; 86:3833-7.

[4] Chomczynski P, Sacchi N. Single-Step method of RNA isolation by acid gunanidinium thiocyanatephenol- chloroform extraction. Anal Biochem 162(1):156-9.

[5] Sambrook J, Fritsch EF, Maniatis T. Molecular Cloning. Cold Spring Harbor Laboratory Press 1989.

[6] Horton RM, Hunt HD, Ho SN, Pullen Jk, et al. Engineering hybrid genes without the use of restriction enzymes: gene splicing by overlap extension. Gene 1989; 77:61-8.

[7] Ji YY, Yang CH, Yeh M. The influence of Trichosanthin on the induction of IgE responses to ovalbumin under adjuvant-free condition. Cell Reasearch 1995; 5:67-74.

[8] Ho SN, Hunt HD, Horton RM, Pullen JK, et al. Site-directed mutagenesis by overlap extension using the polymerase chain reaction. Gene 1989; 77:51-9.

[9] Sparholt SH, Barington T, Schon C. Detection of B-lymphocytes secreting antibodies to Dematophaoides antigens. Clin Exp Allergy 1991; 21:85-9.

Received 27-8-1997. Revised 31-10-1997. Accepted 1-11-1997. 